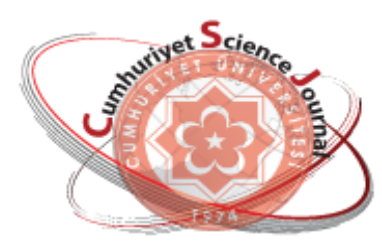

e-ISSN: 2587-246X

ISSN: $2587-2680$

\section{Cumburiyet Scionce Journal \\ esy}

Cumhuriyet Sci. J., Vol.38-4, Supplement (2017) 130-137

\title{
On Some New Ideal Convergent Sequence Spaces of $M_{\lambda}$-Method of Summability
}

\author{
Nazlım Deniz ARAL ${ }^{1 *}$, Şükran KONCA ${ }^{1}$ \\ ${ }^{1}$ Department of Mathematics, Bitlis Eren University, 1300, Bitlis, Turkey
}

Received: 22.06.2017; Accepted: 23.10.2017

http://dx.doi.org/10.17776/csj.363614

\begin{abstract}
In the present work, we introduce some ideal convergent sequence spaces by using $M_{\lambda}$ - summability method which is defined by P. N. Natarajan [On the $\left(M, \lambda_{n}\right)$-method of summability, Analysis] as a typically generalization of Nörlund method. Further, we examine some of their topological properties.
\end{abstract}

Keywords: $I$-convergence, Orlicz function, $M_{\lambda}$-method of summability, sequence space

$M_{\lambda}$ - Toplanabilme Metoduyla Tanımlanan Bazı Yeni İdeal Yakınsak Dizi

\section{Uzayları}

Özet: Bu çalışmada Nörlund metodunun bir genelleşmesi olarak P.N. Natarajan [On the $\left(M, \lambda_{n}\right)$-method of summability, Analysis] tarafından tanımlanan $M_{\lambda}$-toplanabilme metodu kullanılarak bazı ideal yakınsak dizi uzayları tanımlanmıştır. Ayrıca, bu uzayların bazı topolojik özellikleri incelenmiştir.

Anahtar Kelimeler: I -yakınsaklık, Orlicz fonksiyonu, $M_{\lambda}$-toplanabilme metodu, dizi uzay1

\section{INTRODUCTION}

The Orlicz spaces were introduced by Birnbaum and W. Orlicz [1] in 1931. Krasnosel'skii and Rutickii [2] detailed study on Orlicz spaces. Lindberg [3] studied various properties of Orlicz sequence spaces and their subspaces. For further results, see, $[4,5]$.

The notion of $I$-convergence was introduced by Kostyrko, Salat and Wilczynski [6] corresponds to a generalization of the statistical convergence. Related papers can be seen in for example, [7-11].

Natarajan [12] has introduced a very new method of summability which is called $\left(M, \lambda_{n}\right)$ method in 2013 and studied some of its properties concerning its regularity, consistency and translativity. He also has proved an inclusion theorem and an equivalence theorem. Recently, Aral and Küçükaslan [13] have defined $M_{\lambda}$-statistical convergence and given some inclusion results for different $\lambda$ 's, in addition to some relations between statistical convergence and $M_{\lambda}$-statistical convergence given. There are still some open problems on this new method of summability, for example one of them, there have not been any ideal convergent sequence spaces defined yet. In this paper, we have defined some spaces of ideal convergent sequences defined by $M_{\lambda}$-method of summability and Orlicz functions. We also examine some of topological properties of these 
sequence spaces. By this way, we aim to fill this gap.

\section{DEFINITIONS AND PRELIMINARIES}

Before beginning of the presentation of the main results, we recall the following definitions. Throughout the paper, for brevity, by the notation $\lim _{k} x_{k}$ we mean $\lim _{k \rightarrow \infty} x_{k}$ and by $\mathbb{N}, \mathbb{R}$ and $\mathbb{C}$ we mean the set of all natural numbers, the set of all real numbers and the set of all complex numbers respectively. For the convenience, we also use the notation $M_{\lambda}$ instead of $\left(M, \lambda_{n}\right)$ representation given in the work of Natarajan [12].

Definition 1 [6] Let $X \neq \varnothing$ and $P(X)=2^{X}$ be the family of all subsets of $X$. Then, a family of sets $I \subset 2^{X}$ is said to be an ideal on $X$ if and only if $I$ satisfies these conditions:

1. $\varnothing \in I$,

\section{2. $A, B \in I$ imply $A \cup B \in I$,}

3. $A \in I, B \subset A$ imply $B \in I$.

An ideal $I$ is called non-trivial if $I \neq \varnothing$ and $X \notin I$, that is $I \neq 2^{X}$. A non-trivial ideal $I \subset 2^{X}$ is called admissible if $\{x\} \in I$ for each $x \in X$.

Definition 2 [14] Let $X$ be a linear metric space. A function $p: X \rightarrow \mathbb{R}$ is called paranorm, if

1. $p(x) \geq 0$, for all $x \in X$,

2. $p(-x)=p(x)$, for all $x \in X$,

3. $p(x+y) \leq p(x)+p(y)$, for all $x, y \in X$,

4. If $\left(\lambda_{n}\right)$ is a sequence of scalars with $\lambda_{n} \rightarrow \lambda$ as $n \rightarrow \infty$ and $\left(x_{n}\right)$ is a sequence of vectors with $p\left(x_{n}-x\right) \rightarrow 0$ as $n \rightarrow \infty$, then $p\left(\lambda_{n} x_{n}-\right.$ $\lambda x) \rightarrow 0$ as $n \rightarrow \infty$.

Definition 3 [14] An Orlicz function is a function $M:[0, \infty) \rightarrow[0, \infty)$ which is continuous, nondecreasing and convex with $M(0)=0, M(x)>0$ for $x>0$ and $M(x) \rightarrow \infty$ as $x \rightarrow \infty$.
If the convexity of an Orlicz function is replaced by $M(x+y) \leq M(x)+M(y)$, then this function is

called modulus function. If $M$ is an Orlicz function, then $M(\lambda x) \leq \lambda M(x)$ for all $\lambda$ with $0<\lambda<1$. An Orlicz function $M$ is said to satisfy $\Delta_{2}$-condition for all values of $x$, if there exists a constant $K>0$, such that $M(2 x) \leq K M(x)$ for all $x \geq 0$. The $\Delta_{2}$ condition is equivalent to $M(L x) \leq K L M(x)$, for all values of $x>0$ and for $L>1$ [2].

A sequence space $E$ is said to be solid (or normal) if $\left(\alpha_{k} x_{k}\right) \in E$, whenever $\left(x_{k}\right) \in E$, for all sequences $\left(\alpha_{k}\right)$ of scalars with $\left|\alpha_{k}\right| \leq 1$ for all $k \in$ $\mathbb{N}$. It is well known that a sequence space $E$ is normal implies that $E$ is monotone [14].

The following inequality will be used throughout this paper. Let $p=\left(p_{k}\right)$ be a sequence of strictly positive real numbers with $0<p_{k} \leq \sup _{k \in \mathbb{N}} p_{k}=$ $G$, and let $D=\max \left\{1,2^{G-1}\right\}$. Then we have

$\left|a_{k}+b_{k}\right|^{p_{k}} \leq D\left\{\left|a_{k}\right|^{p_{k}}+\left|b_{k}\right|^{p_{k}}\right.$

for all $a_{k}, b_{k} \in \mathbb{C}[15]$.

Let $\lambda=\left(\lambda_{n}\right)$ be a sequence such that $\sum_{n=1}^{\infty}\left|\lambda_{n}\right|<$ $\infty$.

Definition 4 [13] A real valued sequence $x=\left(x_{n}\right)$ is said to be $M_{\lambda}$-convergent to $l \in \mathbb{R}$, called the $M_{\lambda}$-limit of $x$ and denoted by $x_{n} \rightarrow l\left(M_{\lambda}\right)$, if $\sum_{k=0}^{n} \lambda_{n-k} x_{k} \rightarrow l, n \rightarrow \infty$.

\section{Main Results}

Now, we present our main results. Throughout the paper $I$ will be considered as a non-trivial admissible ideal.

Let $M$ be an Orlicz function and let $q=\left(q_{n}\right)$ be a bounded sequence of positive real numbers. We define the following sequence spaces, 


$$
\begin{gathered}
c^{I}(M, q)^{M_{\lambda}}:=\left\{x=\left(x_{n}\right) \in w: I-\lim _{n} M\left(\frac{\left|\sum_{k=0}^{n} \lambda_{n-k} x_{k}-L\right|}{\rho}\right)^{q_{n}}=0 \text { for some } L \text { and } \rho>0\right\}, \\
c_{0}^{I}(M, q)^{M_{\lambda}}:=\left\{x=\left(x_{n}\right) \in w: I-\lim _{n} M\left(\frac{\left|\sum_{k=0}^{n} \lambda_{n-k} x_{k}\right|}{\rho}\right)^{q_{n}}=0 \text { for some } \rho>0\right\}, \\
l_{\infty}(M, q)^{M_{\lambda}}:=\left\{x=\left(x_{n}\right) \in w: \sup _{n \in \mathbb{N}} M\left(\frac{\left|\sum_{k=0}^{n} \lambda_{n-k} x_{k}\right|}{\rho}\right)^{q_{n}}<\infty \text { for some } \rho>0\right\} .
\end{gathered}
$$

We can write

$$
m^{I}(M, q)^{M_{\lambda}}=c^{I}(M, q)^{M_{\lambda}} \cap l_{\infty}(M, q)^{M_{\lambda}} \text { and } m_{0}^{I}(M, q)^{M_{\lambda}}=c_{0}^{I}(M, q)^{M_{\lambda}} \cap l_{\infty}(M, q)^{M_{\lambda}} .
$$

For some special cases we obtain the followings:

1. If $q=\left(q_{n}\right)=1$ for all $n \in \mathbb{N}$, then the sequence spaces given by (2) reduce to the following sequence spaces.

$$
\begin{gathered}
c^{I}(M)^{M_{\lambda}}:=\left\{x=\left(x_{n}\right) \in w: I-\lim _{n} M\left(\frac{\left|\sum_{k=0}^{n} \lambda_{n-k} x_{k}-L\right|}{\rho}\right)=0 \text { for some } L \text { and } \rho>0\right\}, \\
c_{0}^{I}(M)^{M_{\lambda}}:=\left\{x=\left(x_{n}\right) \in w: I-\lim _{n} M\left(\frac{\left|\sum_{k=0}^{n} \lambda_{n-k} x_{k}\right|}{\rho}\right)=0 \text { for some } \rho>0\right\}, \\
l_{\infty}(M)^{M_{\lambda}}:=\left\{x=\left(x_{n}\right) \in w: \sup _{n \in \mathbb{N}} M\left(\frac{\left|\sum_{k=0}^{n} \lambda_{n-k} x_{k}\right|}{\rho}\right)<\infty \text { for some } \rho>0\right\} .
\end{gathered}
$$

2. If we take $M(x)=x$ in (2), then we obtain the followings:

$$
\begin{gathered}
\left(c^{I}\right)^{M_{\lambda}}:=\left\{x=\left(x_{n}\right) \in w: I-\lim _{n}\left(\left|\sum_{k=0}^{n} \lambda_{n-k} x_{k}-L\right|\right)=0 \text { for some } L\right\}, \\
\left(c_{0}^{I}\right)^{M_{\lambda}}:=\left\{x=\left(x_{n}\right) \in w: I-\lim _{n}\left(\left|\sum_{k=0}^{n} \lambda_{n-k} x_{k}\right|\right)=0\right\} \\
\left(l_{\infty}\right)^{M_{\lambda}}:=\left\{x=\left(x_{n}\right) \in w: \sup _{n \in \mathbb{N}}\left(\left|\sum_{k=0}^{n} \lambda_{n-k} x_{k}\right|\right)<\infty\right\} .
\end{gathered}
$$

3. When we take $M(x)=x$ and $q=\left(q_{n}\right)=1$ for all $n \in \mathbb{N}$ in the equation (2) then we have

$$
\begin{gathered}
c^{I}(q)^{M_{\lambda}}:=\left\{x=\left(x_{n}\right) \in w: I-\lim _{n}\left(\left|\sum_{k=0}^{n} \lambda_{n-k} x_{k}-L\right|^{q_{n}}\right)=0 \text { for some } L\right\}, \\
c_{0}^{I}(q)^{M_{\lambda}}:=\left\{x=\left(x_{n}\right) \in w: I-\lim _{n}\left(\left|\sum_{k=0}^{n} \lambda_{n-k} x_{k}\right|^{q_{n}}\right)=0\right\}, \\
l_{\infty}(q)^{M_{\lambda}}:=\left\{x=\left(x_{n}\right) \in w: \sup _{n \in \mathbb{N}}\left(\left|\sum_{k=0}^{n} \lambda_{n-k} x_{k}\right|^{q_{n}}\right)<\infty\right\} .
\end{gathered}
$$

4. Let $p=\left(p_{n}\right)$ be a sequence of positive natural numbers and $P_{n}=\sum_{k=0}^{n} p_{k} \rightarrow \infty$. Take into consider $\lambda=\left(\lambda_{n}\right)$ as

$$
\lambda_{n}=\left(\frac{p_{0}}{P_{n}}, \frac{p_{1}}{P_{n}}, \ldots, \frac{p_{n}}{P_{n}}, 0,0, \ldots\right)
$$


then $M_{\lambda}$-convergence coincide with $N_{p}$-convergence and the sequence spaces given by (2) reduces to the following sequence spaces for $q=\left(q_{n}\right)=1(\forall n \in \mathbb{N})$ and $M(x)=x$;

$$
\begin{gathered}
\left(c^{I}\right)^{N_{p}}:=\left\{x=\left(x_{n}\right) \in w: I-\lim _{n}\left(\left|\frac{1}{P_{n}} \sum_{k=0}^{n} x_{k}-L\right|\right)=0 \text { for some } L\right\}, \\
\left(c_{0}^{I}\right)^{N_{p}}:=\left\{x=\left(x_{n}\right) \in w: I-\lim _{n}\left(\left|\frac{1}{P_{n}} \sum_{k=0}^{n} x_{k}\right|\right)=0\right\}, \\
\left(l_{\infty}\right)^{N_{p}}:=\left\{x=\left(x_{n}\right) \in w: \sup _{n \in \mathbb{N}}\left(\left|\frac{1}{P_{n}} \sum_{k=0}^{n} x_{k}\right|\right)<\infty\right\} .
\end{gathered}
$$

Theorem 1 Let $M$ be an Orlicz function and let $q=\left(q_{n}\right)$ be a bounded sequence of positive real numbers. Then, the spaces $c^{I}(M, q)^{M_{\lambda}}, c_{0}^{I}(M, q)^{M_{\lambda}}, m^{I}(M, q)^{M_{\lambda}}$ and $m_{0}^{I}(M, q)^{M_{\lambda}}$ are linear.

Proof. Let $x, y \in c^{I}(M, q)^{M_{\lambda}}$. Then, there exist positive numbers $\rho_{1}$ and $\rho_{2}$ such that

$$
\begin{aligned}
& I-\lim _{n} M\left(\frac{\left|\sum_{k=0}^{n} \lambda_{n-k} x_{k}-L_{1}\right|}{\rho_{1}}\right)^{q_{n}}=0, \text { for some } L_{1} \in \mathbb{C}, \\
& I-\lim _{n} M\left(\frac{\left|\sum_{k=0}^{n} \lambda_{n-k} y_{k}-L_{2}\right|}{\rho_{2}}\right)^{q_{n}}=0, \text { for some } L_{2} \in \mathbb{C} .
\end{aligned}
$$

For a given $\varepsilon>0$, we have

$$
K_{1}=\left\{k \in \mathbb{N}: M\left(\frac{\left|\sum_{k=0}^{n} \lambda_{n-k} x_{k}-L_{1}\right|}{\rho_{1}}\right)^{q_{n}}\right\}, \quad K_{2}=\left\{k \in \mathbb{N}: M\left(\frac{\left|\sum_{k=0}^{n} \lambda_{n-k} y_{k}-L_{2}\right|}{\rho_{2}}\right)^{q_{n}}\right\}
$$

Let $\rho_{3}=\max \left\{2|\alpha| \rho_{1}, 2|\beta| \rho_{2}\right\}$ and let $\alpha, \beta \in \mathbb{C}$ be scalars. Since $M$ is non-decreasing convex function, so by using inequality (1), we have

$$
\begin{gathered}
\lim _{n} M\left(\frac{\left|\sum_{k=0}^{n} \lambda_{n-k}\left(\alpha x_{k}+\beta y_{k}\right)-\left(\alpha L_{1}+\beta L_{2}\right)\right|}{\rho_{3}}\right)^{q_{n}} \\
\leq \lim _{n} M\left(\frac{|\alpha|\left|\sum_{k=0}^{n} \lambda_{n-k} x_{k}-L_{1}\right|}{\rho_{3}}+\frac{|\beta|\left|\sum_{k=0}^{n} \lambda_{n-k} y_{k}-L_{2}\right|}{\rho_{3}}\right)^{q_{n}} \\
\leq \lim _{n} M\left(\frac{\left|\sum_{k=0}^{n} \lambda_{n-k} x_{k}-L_{1}\right|}{\rho_{1}}\right)^{q_{n}}+\lim _{n} M\left(\frac{\left|\sum_{k=0}^{n} \lambda_{n-k} y_{k}-L_{2}\right|}{\rho_{2}}\right)^{q_{n}} .
\end{gathered}
$$

We have from (3),

$$
\left\{k \in \mathbb{N}: \lim _{n} M\left(\frac{\left|\sum_{k=0}^{n} \lambda_{n-k}\left(\alpha x_{k}+\beta y_{k}\right)-\left(\alpha L_{1}+\beta L_{2}\right)\right|}{\rho_{3}}\right)^{q_{n}}\right\} \subset K_{1} \cup K_{2} .
$$

Hence, $\alpha x+\beta y \in c^{I}(M, q)^{M_{\lambda}}$. Thus $c^{I}(M, q)^{M_{\lambda}}$ is a linear space. We can prove that $c_{0}^{I}(M, q)^{M_{\lambda}}$, $m^{I}(M, q)^{M_{\lambda}}$ and $m_{0}^{I}(M, q)^{M_{\lambda}}$ are linear spaces with similar techniques.

Theorem 2 Let $M$ be an Orlicz function. Then, $c_{0}^{I}(M, q)^{M_{\lambda}} \subset c^{I}(M, q)^{M_{\lambda}} \subset l_{\infty}(M, q)^{M_{\lambda}}$. 
Proof. The inclusion $c_{0}^{I}(M, q)^{M_{\lambda}} \subset c^{I}(M, q)^{M_{\lambda}}$ is obvious. Let $x \in c^{I}(M, q)^{M_{\lambda}}$. Then, there exist $L \in \mathbb{C}$ and $\rho>0$ such that

$$
I-\lim _{n} M\left(\frac{\left|\sum_{k=0}^{n} \lambda_{n-k} x_{k}-L\right|}{\rho}\right)^{q_{n}}=0
$$

We have

$$
M\left(\frac{\left|\sum_{k=0}^{n} \lambda_{n-k} x_{k}\right|}{2 \rho}\right)^{q_{n}} \leq \frac{1}{2} M\left(\frac{\left|\sum_{k=0}^{n} \lambda_{n-k} x_{k}-L\right|}{\rho}\right)^{q_{n}}+\frac{1}{2} M\left(\frac{|L|}{\rho}\right)^{q_{n}}
$$

If we take supremum over $n$ on both sides, we get $x \in l_{\infty}(M, q)^{M_{\lambda}}$. Hence we obtain

$$
c_{0}^{I}(M, q)^{M_{\lambda}} \subset c^{I}(M, q)^{M_{\lambda}} \subset l_{\infty}(M, q)^{M_{\lambda}} .
$$

This completes the proof of the theorem.

Theorem 3 Let $M$ be an Orlicz function and let $q=\left(q_{n}\right)$ be a bounded sequence of positive real numbers. Then, $l_{\infty}(M, q)^{M_{\lambda}}$ is a paranormed space with paranorm defined by

$$
g(x)=\inf _{n \geq 1}\left\{\rho^{\frac{q_{n}}{H}}>0: \sup _{n \in \mathbb{N}} M\left(\frac{\left|\sum_{k=0}^{n} \lambda_{n-k} x_{k}\right|}{\rho}\right)^{q_{n}} \leq 1 \text { for some } \rho>0\right\},
$$

where $H=\max \left\{1, \sup _{n} q_{n}\right\}$.

Proof. It is clear that $g(x)=g(-x)$. Since $M(0)=0$, we get $g(\theta)=0$. Let us take $x, y \in l_{\infty}(M, q)^{M_{\lambda}}$ and denote

$$
K(x)=\left\{\rho>0: \sup _{n} M\left(\frac{\left|\sum_{k=0}^{n} \lambda_{n-k} x_{k}\right|}{\rho}\right)^{q_{n}} \leq 1\right\} \text { and } K(y)=\left\{\rho>0: \sup _{n} M\left(\frac{\left|\sum_{k=0}^{n} \lambda_{n-k} y_{k}\right|}{\rho}\right)^{q_{n}} \leq 1\right\}
$$

Let $\rho_{1} \in K(x)$ and $\rho_{2} \in K(y)$. If $\rho=\rho_{1}+\rho_{2}$, then we have

$$
\sup _{n \in \mathbb{N}} M\left(\frac{\left|\sum_{k=0}^{n} \lambda_{n-k}\left(x_{k}+y_{k}\right)\right|}{\rho}\right) \leq\left(\frac{\rho_{1}}{\rho_{1}+\rho_{2}}\right) \sup _{n \in \mathbb{N}} M\left(\frac{\left|\sum_{k=0}^{n} \lambda_{n-k} x_{k}\right|}{\rho_{1}}\right)+\left(\frac{\rho_{2}}{\rho_{1}+\rho_{2}}\right) \sup _{n \in \mathbb{N}} M\left(\frac{\left|\sum_{k=0}^{n} \lambda_{n-k} y_{k}\right|}{\rho_{2}}\right)
$$

which in terms give us, $\sup _{n \in \mathbb{N}} M\left(\frac{\left|\sum_{k=0}^{n} \lambda_{n-k}\left(x_{k}+y_{k}\right)\right|}{\rho_{1}+\rho_{2}}\right)^{q_{n}} \leq 1$ and

$$
\begin{aligned}
g(x+y) & =\inf \left\{\left(\rho_{1}+\rho_{2}\right)^{\frac{q_{n}}{H}}>0: \rho_{1} \in K(x), \rho_{2} \in K(y)\right\} \\
\leq & \inf \left\{\left(\rho_{1}\right)^{\frac{q_{n}}{H}}>0: \rho_{1} \in K(x)\right\}+\inf \left\{\left(\rho_{2}\right)^{\frac{q_{n}}{H}}>0: \rho_{2} \in K(y)\right\} \\
= & g(x)+g(y) .
\end{aligned}
$$

Let $\left(\sigma^{s}\right)$ be a sequence of scalars with $\sigma^{s} \rightarrow \sigma$, where $\sigma, \sigma^{s} \in \mathbb{C}$ and let $\left(x^{s}\right), x \in l_{\infty}(M, q)^{M_{\lambda}}$ be such that $g\left(x^{s}-x\right) \rightarrow 0$ as $s \rightarrow \infty$. To prove that $g\left(\sigma^{s} x^{s}-\sigma s\right) \rightarrow 0$ as $s \rightarrow \infty$. Let 
$A=\left\{\rho_{s}>0: \sup _{n \in \mathbb{N}} M\left(\frac{\left|\sum_{k=0}^{n} \lambda_{n-k} x^{s}\right|}{\rho_{s}}\right)^{q_{n}} \leq 1\right\}$ and $B=\left\{\rho_{s}^{\prime}>0: \sup _{n \in \mathbb{N}} M\left(\frac{\left|\sum_{k=0}^{n} \lambda_{n-k}\left(x^{s}-x\right)\right|}{\rho_{s}^{\prime}}\right)^{q_{n}} \leq 1\right\}$.

If $\rho_{s} \in A$ and $\rho_{s}^{\prime} \in B$, then we observe that

$$
\begin{gathered}
M\left(\frac{\left|\sum_{k=0}^{n} \lambda_{n-k}\left(\sigma^{s} x^{s}-\sigma x\right)\right|}{\rho_{s}\left|\sigma^{s}-\sigma\right|+\rho_{s}^{\prime}|\sigma|}\right) \leq M\left(\frac{\left|\sum_{k=0}^{n} \lambda_{n-k}\left(\sigma^{s} x^{s}-\sigma x^{s}\right)\right|}{\rho_{s}\left|\sigma^{s}-\sigma\right|+\rho_{s}^{\prime}|\sigma|}+\frac{\left|\sum_{k=0}^{n} \lambda_{n-k}\left(\sigma x^{s}-\sigma x\right)\right|}{\rho_{s}\left|\sigma^{s}-\sigma\right|+\rho_{s}^{\prime}|\sigma|}\right) \\
\quad \leq \frac{\left|\sigma^{s}-\sigma\right| \rho_{s}}{\rho_{s}\left|\sigma^{s}-\sigma\right|+\rho_{s}^{\prime}|\sigma|} M\left(\frac{\left|\sum_{k=0}^{n} \lambda_{n-k} x^{s}\right|}{\rho_{s}}\right)+\frac{|\sigma| \rho_{s}^{\prime}}{\rho_{s}\left|\sigma^{s}-\sigma\right|+\rho_{s}^{\prime}|\sigma|} M\left(\frac{\left|\sum_{k=0}^{n} \lambda_{n-k}\left(x^{s}-x\right)\right|}{\rho_{s}^{\prime}}\right) .
\end{gathered}
$$

From the above inequality, it follows that

$$
\sup _{n \in \mathbb{N}} M\left(\frac{\left|\sum_{k=0}^{n} \lambda_{n-k}\left(\sigma^{s} x^{s}-\sigma x\right)\right|}{\rho_{s}\left|\sigma^{s}-\sigma\right|+\rho_{s}^{\prime}|\sigma|}\right)^{q_{n}} \leq 1
$$

and consequently,

$$
\begin{aligned}
& g\left(\sigma^{s} x^{s}-\sigma x\right) \leq \inf \left\{\left(\rho_{s}\left|\sigma^{s}-\sigma\right|+\rho_{s}^{\prime}|\sigma|\right)^{\frac{q_{n}}{H}}>0: \rho_{s} \in A, \rho_{s}^{\prime} \in B\right\} \\
& \leq\left(\left|\sigma^{s}-\sigma\right|\right)^{\frac{q_{n}}{H}} \inf \left\{\left(\rho_{s}\right)^{\frac{q_{n}}{H}}>0: \rho_{s} \in A\right\}+(|\sigma|)^{\frac{q_{n}}{H}} \inf \left\{\left(\rho_{s}^{\prime}\right)^{\frac{q_{n}}{H}}>0: \rho_{s}^{\prime} \in B\right\} \\
& \leq \max \left\{1,\left|\sigma^{s}-\sigma\right|^{\frac{q_{n}}{H}}\right\} g\left(x^{s}\right)+\max \left\{1,|\sigma|^{\frac{q_{n}}{H}}\right\} g\left(x^{s}-x\right) .
\end{aligned}
$$

Hence by our assumption, the right hand side tends to 0 as $s \rightarrow \infty$. This completes the proof.

Theorem 4 Let $M_{1}$ and $M_{2}$ be Orlicz functions that satisfy the $\Delta_{2}$-condition. Then, for $Z=c^{I}, c_{0}^{I}, m^{I}, m_{0}^{I}$,

1. $Z\left(M_{2}, q\right)^{M_{\lambda}} \subseteq Z\left(M_{1} \circ M_{2}\right)^{M_{\lambda}}$,

2. $Z\left(M_{1}, q\right)^{M_{\lambda}} \cap Z\left(M_{2}, q\right)^{M_{\lambda}} \subseteq Z\left(M_{1}+M_{2}, q\right)^{M_{\lambda}}$.

Proof. (1) Let $x \in c_{0}^{I}(M, q)^{M_{\lambda}}$. Then, there exist $\rho>0$ such that

$$
I-\lim _{n} M_{2}\left(\frac{\sum_{k=0}^{n} \lambda_{n-k} x_{k}}{\rho}\right)^{q_{n}}=0 .
$$

Let $\varepsilon>0$ and choose $\delta$ with $0<\delta<1$ such that $M_{1}(t)<\varepsilon$ for $0 \leq t \leq \delta$. Write $y_{n}=$ $M_{2}\left(\frac{\left|\sum_{k=0}^{n} \lambda_{n-k} x_{k}\right|}{\rho}\right)^{q_{n}}$ and consider $\lim _{n \in \mathbb{N}} M_{1}\left(y_{n}\right)=\lim _{\substack{y_{n} \leq \delta \\ n \in \mathbb{N}}} M_{1}\left(y_{n}\right)+\lim _{\substack{y_{n}>\delta \\ n \in \mathbb{N}}} M_{1}\left(y_{n}\right)$. Since $M_{1}$ is an Orlicz function, we have

$$
\lim _{\substack{y_{n} \leq \delta \\ n \in \mathbb{N}}} M_{1}\left(y_{n}\right) \leq M_{1}(2) \lim _{\substack{y_{n} \leq \delta \\ n \in \mathbb{N}}}\left(y_{n}\right)
$$

For $y_{n}>\delta$ we have $y_{n}<\frac{y_{n}}{\delta}<1+\frac{y_{n}}{\delta}$. 
Since $M_{1}$ is nondecreasing and convex, it follows that

$$
M_{1}\left(y_{n}\right)<M_{1}\left(1+\frac{y_{n}}{\delta}\right)<\frac{1}{2} \frac{M_{1}\left(2 y_{n}\right)}{\delta}
$$

Since $M_{1}$ satisfies $\Delta_{2}$-condition we have

$$
M_{1}\left(y_{n}\right)<\frac{1}{2} K \frac{y_{n}}{\delta} M_{1}(2)+\frac{1}{2} K \frac{y_{n}}{\delta} M_{1}(2)=K \frac{y_{n}}{\delta} M_{1}(2) .
$$

Hence,

$$
\lim _{\substack{y_{n}>\delta \\ n \in \mathbb{N}}} M_{1}\left(y_{n}\right) \leq \max \left(1, K \delta^{-1} M_{1}(2)\right) \lim _{\substack{y_{n}>\delta \\ n \in \mathbb{N}}}\left(y_{n}\right)
$$

From (4), (5) and (6), we have $x=\left(x_{k}\right) \in c_{0}^{I}\left(M_{1} \circ M_{2}, q\right)^{M_{\lambda}}$. Thus $c_{0}^{I}\left(M_{2}, q\right)^{M_{\lambda}} \subseteq c_{0}^{I}\left(M_{1} \circ M_{2}, q\right)^{M_{\lambda}}$.

We can prove the other cases similarly.

(2) Let $x \in c_{0}^{I}\left(M_{1}, q\right)^{M_{\lambda}} \cap c_{0}^{I}\left(M_{2}, q\right)^{M_{\lambda}}$ then there exist $\rho>0$ such that

$$
I-\lim _{n} M_{1}\left(\frac{\left|\sum_{k=0}^{n} \lambda_{n-k} x_{k}\right|}{\rho}\right)^{q_{n}}=0 \text { and } I-\lim _{n} M_{2}\left(\frac{\left|\sum_{k=0}^{n} \lambda_{n-k} x_{k}\right|}{\rho}\right)^{q_{n}}=0 .
$$

The result is obtained by the following equality

$$
\lim _{n \in \mathbb{N}}\left(M_{1}+M_{2}\right)\left(\frac{\left|\sum_{k=0}^{n} \lambda_{n-k} x_{k}\right|}{\rho}\right)^{q_{n}}=\lim _{n \in \mathbb{N}} M_{1}\left(\frac{\left|\sum_{k=0}^{n} \lambda_{n-k} x_{k}\right|}{\rho}\right)^{q_{n}}+\lim _{n \in \mathbb{N}} M_{2}\left(\frac{\left|\sum_{k=0}^{n} \lambda_{n-k} x_{k}\right|}{\rho}\right)^{q_{n}}
$$

Corollary 1 Let $M$ be an Orlicz function which satisfies $\Delta_{2}$-condition. Then $Z(q)^{M_{\lambda}} \subseteq Z(M, q)^{M_{\lambda}}$ holds for $Z=c^{I}, c_{0}^{I}, m^{I}, m_{0}^{I}$.

Theorem 5 The spaces $c_{0}^{I}(M, q)^{M_{\lambda}}$ and $m_{0}^{I}(M, q)^{M_{\lambda}}$ are solid.

Proof. We will prove for the space $c_{0}^{I}(M, q)^{M_{\lambda}}$. For $m_{0}^{I}(M, q)^{M_{\lambda}}$, the proof shall be similar. Let $x \in$ $c_{0}^{I}(M, q)^{M_{\lambda}}$, then there exists $\rho>0$ such that

$$
I-\lim _{n} M\left(\frac{\left|\sum_{k=0}^{n} \lambda_{n-k} x_{k}\right|}{\rho}\right)^{q_{n}}=0 .
$$

Let $\alpha_{k}$ be a sequence of scalars such that $\left|\alpha_{k}\right| \leq 1, \forall k \in \mathbb{N}$. Then the result follows from the following inequality

$$
\lim _{n} M\left(\frac{\left|\sum_{k=0}^{n} \lambda_{n-k} \alpha_{k} x_{k}\right|}{\rho}\right)^{q_{n}} \leq \lim _{n} M\left(\frac{\left|\sum_{k=0}^{n} \lambda_{n-k} x_{k}\right|}{\rho}\right)^{q_{n}}=0
$$

and this completes the proof.

Corollary 2 The spaces $c_{0}^{I}(M, q)^{M_{\lambda}}$ and $m_{0}^{I}(M, q)^{M_{\lambda}}$ are monotone. 


\section{REFERENCES}

[1]. Birnbaum Z., Orlicz W. Uber die Verallgemeinerung des Begriffes der zueinander Konjugierten Potenzen, Studia Mathematica, 1931; 3: 1-67.

[2]. Krasnosel'skii M.A., Rutickii Y.B. Convex Functions and Orlicz Spaces, P. Noordhoff Ltd., Groningen, Netherlands, 1961.

[3]. Lindberg, K., On subspaces of Orlicz sequence spaces, Studia Mathematica, 1973; 45 (2):119-146.

[4]. Lindenstrauss J., Tzafriri L. On Orlicz Sequence Spaces, Israel Journal of Mathematics, 1971; 10 (3): 379-390.

[5]. Parashar S.D., Choudhary B. Sequence Spaces Defined by Orlicz Function, Indian Journal Pure and Applied Mathematics, 1994; 24 (4): 419-428.

[6]. Kostyrko P., Wilczynski W., Salat T. Iconvergence, Real Analysis Exchange, 2000; 26 (2): 669-686.

[7]. Tripathy B.C., Hazarika B. Some Iconvergent sequence spaces defined by Orlicz functions, Acta Mathematicae Applicatae Sinica, 2011; 27 (1): 149-154.
[8]. Savas E. On some new sequence spaces in 2-normed spaces using ideal convergence and an Orlicz function, Journal of Inequalities and Applications, 2010; Article ID 482392, 8 pages.

[9]. Mursaleen M., Sharma S.K. Spaces of Ideal Sequences, Hindawi, 2014; Artical ID 134534, 6 pages.

[10]. Savaş E., Das P. A generalized statistical convergence via ideals, Application Letters, 2011; 24 (6): 826-830.

[11]. Salat T., Tripathy B.C., Ziman M. On some properties of I-convergence, Tatra Mountains Mathematical Publications, 2004; 28: 279-286.

[12]. Natarajan P.N. On the $\left(M, \lambda_{n}\right)$ method of summability, Analysis, 2013; \{33\}: 51-56.

[13]. Aral N.D., Küçükaslan M. On $M_{\lambda}$ statistical Convergence, Journal of Mathematical Analysis, 2016; 7 (2): 37-46.

[14]. Kamrhan P.K., Gupta M. Sequence spaces and series Marcel Dekker, New York, 1981.

[15]. Maddox I.J. Elements of Functional Analysis Cambridge Univ. Press, Cambridge, 1970. 